\title{
Using computer-assisted demonstrations of optical phenomena in an undergraduate optics course
}

John Tarvin, Stephen Cobb, Louis Beyer

John T. Tarvin, Stephen H. Cobb, Louis M. Beyer, "Using computer-assisted demonstrations of optical phenomena in an undergraduate optics course," Proc. SPIE 2525, 1995 International Conference on Education in Optics, (13 October 1995); doi: 10.1117/12.224022

Event: SPIE's 1995 International Symposium on Optical Science, Engineering, and Instrumentation, 1995, San Diego, CA, United States 


\title{
USING COMPUTER-ASSISTED DEMONSTRATIONS OF OPTICAL PHENOMENA IN AN UNDERGRADUATE OPTICS COURSE
}

\author{
John T. Tarvin \\ Department of Physics \\ Samford University, Birmingham, AL 35229 \\ Stephen H. Cobb and Louis M. Beyer \\ Department of Physics and Astronomy \\ Murray State University, Murray, KY 42071
}

\begin{abstract}
A set of computer programs has been developed for the visual presentation of introductory optical phenomena. These computer simulations were created to serve a dual purpose: as demonstration aids in an NSF-sponsored Optics Demonstration Laboratory, and as teaching aids in undergraduate geometrical and physical optics courses. In the field of diffractive optics, simulations include the calculation of intensity patterns for unobscured and obscured apertures in both rectangular and circular geometries. These patterns can be compared to those measured in the laboratory with a CCD camera. A program for calculating the diffraction pattern for a two-dimensional aperture of arbitrary shape has also been developed. These programs, when coordinated with homework assignments, allow students to compare their theoretical derivations with a correct numerical solution for the same problem. In the field of geometrical optics, a ray-trace program appropriate for gradient-index fibers with cylindrical symmetry has been developed. This program enables the student to study the focusing properties of such fibers, and to predict how such properties depend on the index profile and on the length of the optical fiber. Examples of these programs will be presented, along with a report on the success of these programs as a vehicle for imparting a conceptual understanding of the physical principles involved.
\end{abstract}

Keywords: undergraduate optics, computer-assisted demonstrations, obscured apertures, unobscured apertures, gradient-index fibers, GRIN

\section{INTRODUCTION}

Computer simulation and demonstration programs have become increasingly popular during the past several years. In fact, several current introductory physics texts now offer integrated software packaged with the text. ${ }^{1,23}$ In most cases, this simulation software is concentrated in the area of classical mechanics - which can include the motion of charged particles - and thermodynamics. Optical demonstrations, where available, are usually limited to elementary topics in geometrical optics.

The purpose of the present report is to describe several related demonstration programs in the areas of both physical optics and geometrical optics. These programs use high-resolution displays and an interactive interface to demonstrate optical principles. One set of programs deals with diffractive 
optics; these programs were developed to compare CCD-acquired images from both obscured and unobscured circular apertures with theoretical predictions. A third program calculated the diffraction pattern, generated by a rectangular grid of sources in the aperture plane, on a rectangular grid of points in the image plane. A second set of programs deals with ray propagation through a gradient-index (GRIN) medium. The motivation for these programs was an experimental article in which GRIN rods were used to construct a retroreflector. ${ }^{4}$ Two of the authors (JTT and SHC) have previously reported on the use of animated computer simulations in other areas of physical optics. ${ }^{5}$

\section{PROGRAM DESCRIPTION}

A brief description of each of the demonstration programs will be presented in the following sections. All programs run under the MS DOS operating system; they will also run under a DOS session using WINDOWS 3.x. The programs are coded in either C, FORTRAN, or BASIC, depending on the program. The authors can be contacted for information about program availability.

\subsection{Diffraction programs}

The diffraction program for circular apertures is built around the need to display a diffraction pattern experimentally-acquired with a CCD camera. This image is displayed using a 64-level grey-scale

palette. A cursor can be moved across the image using arrow keys. The intensity profiles of the row and column defining the cursor location are also plotted on the screen; the gain of these profiles is under user control, so small details can be enhanced. Estimates for the image peak amplitude, aperture diameter, obscuration diameter and pixel location, and screen-to-aperture distance can be entered by the user. The resulting theoretical intensity prediction ${ }^{6}$ corresponding to these parameters is calculated and overlaid on the intensity profiles mentioned above. When satisfied that the initial estimated are "close," the program can be instructed to perform a non-linear least-squares fit to the data using the current estimates as starting guesses. Separate programs are used for obscured and unobscured apertures; these programs differ only in the inclusion or exclusion, respectively, of obscuration parameters.

A program that displays grey-scale intensity pattern for an arbitrary rectangular grid of source points in the aperture plane has also been developed. The calculation method used in this program is based on that of Rudolf, et al. ${ }^{7}$ This program is used as a demonstration program for apertures of arbitrary shape. The program interface is similar to the above programs, with the exception that there is no provision for viewing experimental images or for performing a fit.

\subsection{GRIN programs}

The GRIN programs are designed to show an animated ray trace through a GRIN rod having axial symmetry and a quadratic index variation. The ray trace is an implementation of the method of Sharma, et $\mathrm{al}^{8}{ }^{8}$ The diameter and length of the rod, as well as the initial direction and radial location of the incident ray, are user selectable. GRIN parameters appropriate for commercially-available rods are used. ${ }^{4}$ There are two versions of this program, differing only in whether the far end of the rod is reflective or not. 


\section{PROGRAM USAGE}

The above programs have been used at Murray State University in both a classroom and a laboratory setting. The GRIN programs are used as an introduction to GRIN material and behavior. This material is presented as a special topic in an advanced course in physical optics. It is also used in an upper-level course on the fundamentals of lasers and electro-optics. Students are introduced to the concept of a focal length which is a function of the length of the GRIN rod. Since an exact ray-trace technique is used, they are also able to observe the changes in the focal point for non-paraxial rays. Finally, they can apply these concepts to the fabrication of a retroreflector, and can read about an actual implementation of such a reflector in the literature. ${ }^{4}$

The diffraction programs have been used in the above advanced course in physical optics and in undergraduate research courses. CCD images obtained from circular apertures - both obscured and unobscured - are obtained using a He-Ne laser and various-sized apertures. This data can then be displayed along with best guesses of the aperture diameter, obscuration diameter, image intensity, and camera distance. The direct display of both the raw data and the theoretically-predicted fit for any row or column in the image provides the student with a visual example of how these parameters affect the predicted fit. The rectangular grid program provides students with a good "feel" of the density of source points required to accurately reproduce the pattern for a "known" aperture. This program also gives students an appreciation for the calculational time required to get, for example, one-percent accuracy between calculated and analytic results.

The above programs are going to be used at Samford University in an introductory optics course in the Fall of 1995. This course will be a survey, rather than an upper-level, course; in this case, the programs will be used for special in-class demonstrations.

\section{CONCLUSION}

A description of several optical demonstration programs has been presented. These programs are designed to be used by undergraduate students in either a classroom or a laboratory setting. Initial student feedback has indicated that these programs are accomplishing their goal of providing visualization of various optical phenomena at both the experimental and the theoretical levels. In particular, the introduction of an exact ray-trace solution to propagation through a GRIN rod introduces students to a practical and interesting topic that is often not treated in an introductory optics course.

\section{ACKNOWLEDGMENTS}

The authors gratefully acknowledge the funding support of NSF via III grant USE-9250289. This funding provided for the establishment of an Optics and Laser Demonstration Laboratory at Murray State University; CCD data was obtained through this laboratory. The authors also acknowledge NASA funds made available through the Kentucky Space Grant Consortium for the purchase of a CCD camera. Murray State University College of Science provided additional support in the form of in-kind matches for these grants; this support is gratefully acknowledged. 


\section{REFERENCES}

1. H. C. Ohanian, Principles of Physics, W. W. Norton, New York, 1994.

2. R. A. Serway and J. S. Faughn, College Physics, 4th edition, Saunders, Orlando, 1995.

3. D. C. Giancoli, Physics, 4th edition, Prentice Hall, Englewood Cliffs, 1995.

4. S. Nemoto and J. Kida, "Retroreflector using gradient-index rods," Applied Optics, Vol. 30, No. 7, pp. 815-822, 1991.

5. J. T. Tarvin, S. H. Cobb, and D. L. Jackson, "Demonstrating principles of physical optics through interactive computer simulations," International Conference on Education in Optics, SPIE Vol. 1603, G. B. Altshuler and B. J. Thompson, eds., pp. 358-363, 1991. 1964.

6. M. Born and E. Wolf, Principles of Optics, 2rd edition, pp. 396, 416, Pergamon, New York,

7. P. G. Rudolf, J. J. Tollett, and R. R. McGowan, "Computer modeling wave propagation with a variation of the Helmholtz-Kirchhoff relation," Applied Optics, Vol. 29, No. 7, pp. 998-1003, 1990.

8. A. Sharma, D. V. Kumar, and A. K. Ghatak, "Tracing rays through graded-index media: a new method," Applied Optics, Vol. 21, No. 6, pp. 984-987, 1982. 